\title{
ICCD speckle observations of binary stars: Measurements during 1994-1995
}

\author{
E. Aristidi ${ }^{1}$, M. Carbillet ${ }^{1}$, J.-L. Prieur ${ }^{2}$, B. Lopez ${ }^{3}$, Y. Bresson ${ }^{3}$, and L. Koechlin ${ }^{2}$ \\ 1 UMR 6525 Astrophysique, Université de Nice Sophia, Antipolis, Centre National de la Recherche Scientifique, Parc Valrose, \\ F-06108 Nice Cedex 2, France \\ 2 Observatoire Midi Pyrénées, 14 Av. Edouard Belin, F-31000 Toulouse, France \\ ${ }^{3}$ UMR 6528, Département Fresnel de l'Observatoire de la Côte d'Azur, BP. 4229, F-06304 Nice Cedex 4, France
}

Received March 4; accepted April 1, 1997

\begin{abstract}
We present speckle observations of nineteen double stars and the triple star 2 Cam. Angular separations, absolute position angles and relative photometry result from these observations. The angular separation is derived from the power spectrum. The position angle and the relative photometry are determined by two recent techniques: the cross-correlation between the speckle images and their square, and the ratios of twofold probability density functions of the images.
\end{abstract}

Key words: methods: data analysis - techniques: image processing - techniques: interferometric — binaries: close

\section{Introduction}

This paper presents the results of double star speckle observations performed at the $2 \mathrm{~m}$ Télescope Bernard Lyot (TBL) of Pic du Midi observatory (France). Bright and well-resolved binaries were chosen for the first observing run in 1994. In 1995, we measured long-period double stars for which the published orbit had to be revisited. One of the speckle analysis method used is based upon the computation of probability density functions of the intensity of the specklegrams (Carbillet et al. 1996b). Its major capability is to give an accurate relative photometry of double stars. Our work is the first routine application of this technique developed by the Probability Imaging group of Nice University. Relative photometry computed this way is found to be consistent with direct imaging reconstruction obtained from bispectral techniques. Absolute position angles $(\theta)$ were calculated using a cross-correlation technique (Aristidi et al. 1997).

\footnotetext{
Send offprint requests to: E. Aristidi

* Based on observations made at $2 \mathrm{~m}$ Télescope Bernard Lyot, Pic du Midi, France.
}

This paper is organized as follows: Sect. 2 presents the instrumentation and the list of observed objects together with observing conditions. Section 3 details the data reduction and the speckle methods used. Section 4 summarizes the results and gives a comparison between observed and predicted binary star separations and position angles.

\section{Observations}

Data were recorded during two observing runs in September 1994 and December 1995 at the $2 \mathrm{~m}$ TBL. A total of 20 double stars and a triple star (2 Cam) were observed during five nights. Details are given in Table 1. One star (SAO 12917) was discovered as double during the 1995 run. As its measurement has been already published in a research note (Carbillet et al. 1996a) this star will no more appear in the present paper.

The speckle camera was developed by the Aperture Synthesis group of Observatoire Midi-Pyrénées (André et al. 1994; Prieur et al. 1994). It allows different magnifications of the focal image, has a filter wheel equipped with $B, V, R$ interferential filters and compensates the atmospheric refraction by means of Risley prisms. The speckle camera was put at the $f / 25$ Cassegrain focus of the TBL. The angular field of view was $8^{\prime \prime}$. $\times 11^{\prime \prime}$. for the observations of $1995,33^{\prime \prime} 5 \times 4{ }^{\prime \prime} .5$ for the observations of 1994 .

The detector is an intensified video CCD (Philips IP800T). The images are sampled by a $290 \times 774$ pixels CCD matrix. The effective pixel size at the front of the intensifier is $17.7 \mu \mathrm{m} \times 32.9 \mu \mathrm{m}$. Exposure time ranges from $64 \mu$ s to $16 \mathrm{~ms}$. Video signal is transferred to the observation room by fiber optics. Images are recorded on S-VHS tape using a Panasonic AG-7355. Local processing is made by a NeXT Cube computer with a NeXT Dimension video digitizer. This local processing is far from real-time since the transfer rate is of the order of 2 images per second for a $256 \times 256$ sub-image. Post-processing is made with the 
Table 1. Journal of observations. $\lambda$ denotes the wavelength and $\Delta \lambda$ the bandwidth. $\Delta t$ is the exposure time. The reference star could not be observed for ADS 14787 and ADS 15281 because of clouds

\begin{tabular}{|c|c|c|c|c|c|c|c|}
\hline $\begin{array}{c}\text { ADS } \\
\text { number }\end{array}$ & $\begin{array}{c}\text { Star } \\
\text { name }\end{array}$ & $\begin{array}{c}\text { Reference } \\
\text { star }\end{array}$ & Epoch & $\begin{array}{c}\lambda / \Delta \lambda \\
(\mathrm{nm})\end{array}$ & $\begin{array}{c}\Delta t \\
(\mathrm{~ms})\end{array}$ & $\begin{array}{c}\text { Sampling } \\
(\mathrm{mas} / \text { pixel })\end{array}$ & Notes \\
\hline \hline 434 & $\lambda$ Cas & $\tau$ Cas & 1995.951 & $528 / 69$ & 16 & 30.8 & \\
\hline 1615 & $\alpha$ Psc & $\delta$ Cet & 1995.951 & $528 / 69$ & 16 & 30.8 & 1 \\
\hline 1860 & $\iota$ Cas & SAO 12031 & 1995.951 & $528 / 69$ & 16 & 30.8 & 1,3 \\
\hline 2616 & 7 Tau & SAO 75912 & 1995.951 & $684 / 69$ & 16 & 30.8 & 1,3 \\
\hline $2755 \mathrm{AB}$ & & ADS 2755C & 1995.951 & $684 / 69$ & 16 & 30.8 & 1,3 \\
\hline 3358 & 2 Cam & SAO 24531 & 1995.940 & $684 / 69$ & 11 & 30.8 & \\
\hline 3711 & 14 Ori & SAO 112556 & 1995.951 & $684 / 69$ & 5 & 30.8 & 2,3 \\
\hline 3728 & & SAO 112308 & 1995.951 & $684 / 69$ & 5 & 30.8 & 3 \\
\hline 4115 & 32 Ori & $\omega$ Ori & 1995.930 & $684 / 69$ & 16 & 11.9 & 1,3 \\
\hline 5447 & & SAO 95996 & 1995.951 & $684 / 69$ & 5 & 30.8 & 3 \\
\hline 5925 & & SAO 152663 & 1995.951 & $684 / 69$ & 16 & 30.8 & 3 \\
\hline 7307 & & SAO 42876 & 1995.930 & $684 / 69$ & 16 & 11.9 & 3 \\
\hline 8035 & $\alpha$ Uma & $\beta$ Uma & 1995.940 & $684 / 69$ & 0.4 & 30.8 & \\
\hline 10360 & $c$ Her & 59 Her & 1994.696 & $658 / 42.5$ & 16 & 11.9 & \\
\hline 12973 & $\zeta$ Sge & HR 7536 & 1994.696 & $658 / 42.5$ & 16 & 11.9 & \\
\hline 14073 & $\beta$ Del & $\epsilon$ Del & 1994.693 & $658 / 42.5$ & 16 & 11.9 & \\
\hline 14773 & $\delta$ Equ & $\rho$ Aql & 1994.693 & $658 / 42.5$ & 16 & 11.9 & 3 \\
\hline 14787 & $\tau$ Cyg & - & 1994.693 & $658 / 42.5$ & 13 & 11.9 & \\
\hline 15281 & $\kappa$ Peg & - & 1994.696 & $658 / 42.5$ & 16 & 11.9 & \\
\hline 16057 & & SAO 10131 & 1995.940 & $684 / 69$ & 16 & 30.8 & \\
\hline
\end{tabular}

Notes: 1: Very poor seeing conditions. 2: Bad atmospheric refraction compensation.

3: Faint object, close to photon-counting conditions.

same computer driving the video recorder via a $\mathrm{RS}-232 \mathrm{C}$ line and reading the tape frame by frame.

Each binary star and its reference star were observed about 20 minutes. The reference stars have been chosen of similar magnitude and spectral type as the double stars, to reproduce the same observing conditions for the detector. The limiting magnitude of the experiment is about $V=10$ for medium seeing conditions $\left(F W H M \simeq 1^{\prime \prime} \cdot 2\right)$.

Calibration for the spatial sampling was made by putting a grid into the focal plane. The angular calibration was made by trailing a star across the field in right ascension and declination. The images taken in 1994 were oversampled with 119 mas per pixel, whereas the speckle size was 61 mas at $490 \mathrm{~nm}$ (the shortest wavelength available). In 1995 we used a lower magnification and the pixel size was just half of the speckle size at $490 \mathrm{~nm}$ (Nyquist criterion).

\section{Data reduction}

\subsection{Pre-processing}

A typical number of 6000 specklegrams are read from the video tapes for each object (binary star and reference star). For faint objects (mag $\gtrsim 9$ ) this number can reach 15000. Frames are digitized within a square sub-image of the video field. The size of this sub-image is generally
$128 \times 128$ or $256 \times 256$, depending on the star separation and the size of the long-exposure image. The total size of the video field is $384 \times 288$. Each video grab is then checked for bad points, null images and loss of video synchronization signal. Images showing these features are deleted.

During the observation of a star, seeing conditions may change and images are classified according to the strength of turbulence before processing. This classification is performed the following way. The square modulus of the Fourier transform of each specklegram is computed. An instantaneous Fried parameter $r_{0}$ is estimated by fitting the low-frequencies of this energy spectrum with a Kolmogorov spectrum (Fried 1966; Roddier 1981):

$$
B(f)=\exp \left\{-6.88\left(\lambda f / r_{0}\right)^{5 / 3}\right\}
$$

where $f$ is the spatial frequency and $\lambda$ the wavelength. After each specklegram is associated to a Fried parameter, images are binned into 5 classes according to image quality: class 5 contains the best images (low turbulence, good visual quality), class 1 the worst. Figure 1 gives an illustration of typical frames in each class. The processing of the data is made separately for each class. The classes we have processed are mainly classes 3 and 4 since the number of images belonging to class 5 was often too low $(10-40)$. 

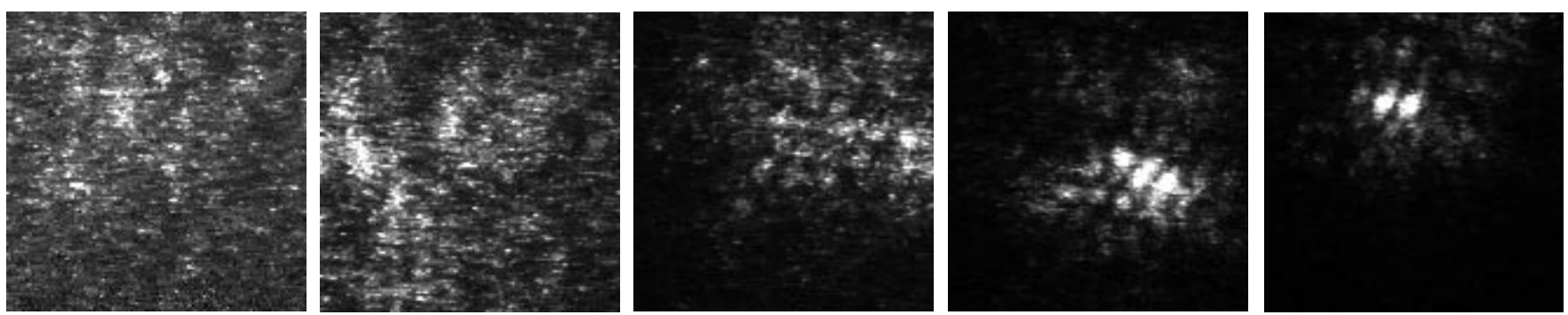

Fig. 1. Classes of specklegrams illustrated with the star $c$ Her. From left to right: classes 1 to 5 . The field of view is $1^{\prime \prime} .5 \times 1^{\prime \prime} 5$. From a total number of 4999 frames of $c$ Her digitized from the tape, the class repartition was the following. Class 1: 468 images, class 2: 2979 images, class 3: 1350 images, class 4: 191 images and class 5: 11 images. On the best images (class 5), the double star can sometimes be seen directly on the specklegrams

\subsection{Separation measurement}

The separation $\rho$ is derived from the autocorrelation function. The power spectrum of both the double star and the reference star are computed for a given image class. The visibility function is obtained by dividing the two power spectra. An apodisation is then made by multiplying the visibility function by a transfer function of a circular telescope. The Fourier transform of the result gives the autocorrelation of the double star corrected from the turbulence. For a binary star, this function shows a central peak and two lateral ones. The distance between the central peak and the lateral ones gives the star separation. In practice we computed the photocenter of the lateral peaks on a $5 \times 5$ grid centered on the peak maximum. The error bar is obtained by differentiation of the formula used for photocenter determination, the noise being estimated in a $10 \times 10$ grid located far from the central and the lateral peaks. The accuracy depends on various parameters such as seeing conditions, magnitude difference, and total magnitude of the binary star and the reference star. The 1994 measurements (oversampled images) were more precise (typical errors 3 mas) than the 1995 ones (typical errors 15 mas).

\subsection{Position angle measurement}

Absolute position angles (i.e. containing the quadrant information) were computed using the recent technique of cross correlation (Aristidi et al. 1996) which is currently under development at the Département d'Astrophysique of Nice University. This technique consists of computing the ensemble average of the cross-correlation between the specklegrams and their square. In practice the computation is made in the Fourier plane: the cross spectrum between the specklegrams and their square is obtained both for the double star and the reference star. It is a complex quantity whose imaginary part contains the absolute quadrant information. The cross-spectrum of the double star is then divided by those of the reference star. An apodizing function (transfer function of a circular telescope) is applied and a Fourier transform is made. Although the result looks very similar to the autocorrelation (a central peak and two lateral ones), the lateral peaks of the cross-correlation are asymmetric. As presented in Fig. 2 for several binaries, this asymmetry gives the absolute position of the companion.

\subsection{Relative photometry measurement}

The relative photometry of the system is obtained by using ratios of twofold probability density functions (Carbillet et al. 1996b). The twofold probability density function (PDF) is a function of two random variables of intensity $\left(\Omega_{1}\right.$ and $\left.\Omega_{2}\right)$, and a space-lag $\boldsymbol{r}$. Whatever the value of $\boldsymbol{r}$, the twofold PDF for a point-source (a non-resolved reference star) has a symmetrical structure in $\Omega_{1}$ and $\Omega_{2}$. For $\boldsymbol{r}$ close to the separation vector $\boldsymbol{\rho}$ between the two components, the twofold PDF for a binary star has an arrowhead shape with a trend towards a direction $\Omega_{2}$ of the order of $\alpha \Omega_{1}$, where $\alpha$ is the oriented intensity ratio. The direction $\alpha \Omega_{1}$ is tremendously enhanced by dividing the twofold PDF of the binary star, computed for $\boldsymbol{r}=\boldsymbol{\rho}$, by the twofold PDF of the reference star, computed for the same space-lag $\boldsymbol{\rho}$. The resulting quantity, the so-called function $Q$, clearly shows a ridge that simply follows: $\Omega_{2}=\alpha \Omega_{1}$. The oriented intensity ratio $\alpha$ can then be easily evaluated by doing a radial integration of $Q\left(\Omega_{1}, \Omega_{2}\right): I_{Q}(\theta)$, where $\theta$ is the angle measured in polar coordinates in the $\left(\Omega_{1}, \Omega_{2}\right)$ plane. The function $Q-Q^{T}$, where $Q^{T}$ is the transposed quantity of $Q$, can also be computed in order to enhance the relevant ridge. In that case, the radial integration gives both $\alpha$ (corresponding to the maximum of $I_{Q-Q^{T}}$ ), and $1 / \alpha$ (corresponding to the minimum of $I_{Q-Q^{T}}$ ). The error bars are then derived from a binomial fit of the regions close to the extrema of the radial integration, since a convex function has a quadratic behavior close to its extrema.

An alternative quantity may be used when no reference star is available: the division of the twofold PDF computed for $\boldsymbol{r}=\boldsymbol{\rho}$ by the twofold PDF computed for $\boldsymbol{r} \perp \boldsymbol{\rho}$.

Figure 3 shows the functions $Q-Q^{T}$ (and their radial integrations) computed for three different type of data. 

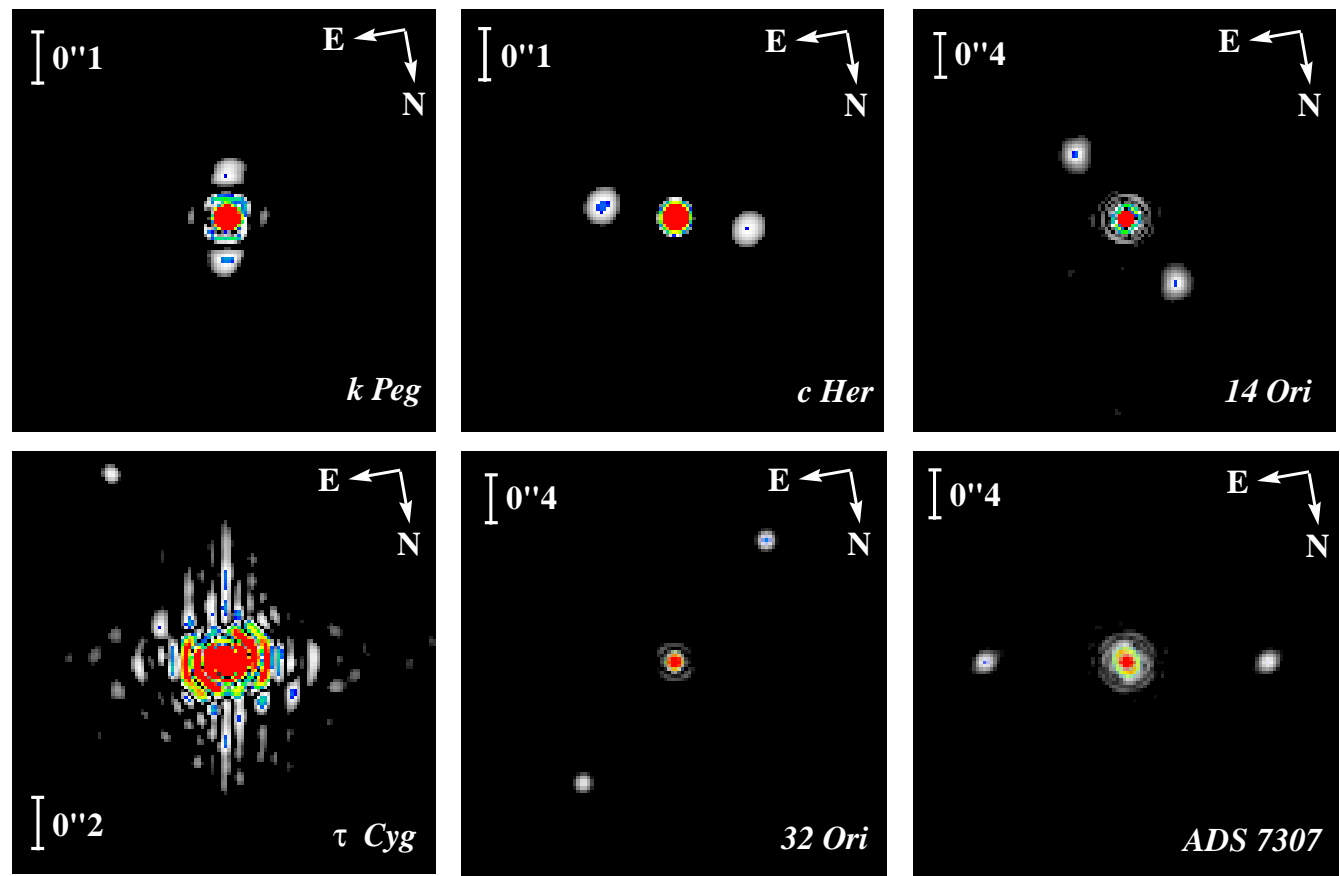

Fig. 2. Gray-level plots of cross-correlations computed on the specklegrams of six binary stars. This function shows a central peak surrounded by two smaller asymmetric ones. The asymmetry of the secondary peaks gives the orientation of the couple, the distance between lateral peaks and the central one provides the angular separation. The look-up table used for this representation is drawn on the right. Scale and orientation are indicated at the top of each picture. The case of $\tau$ Cyg is interesting: due to the large magnitude difference of the couple $(\Delta m=3)$, only one of the lateral peaks is visible, the second one being in the noise
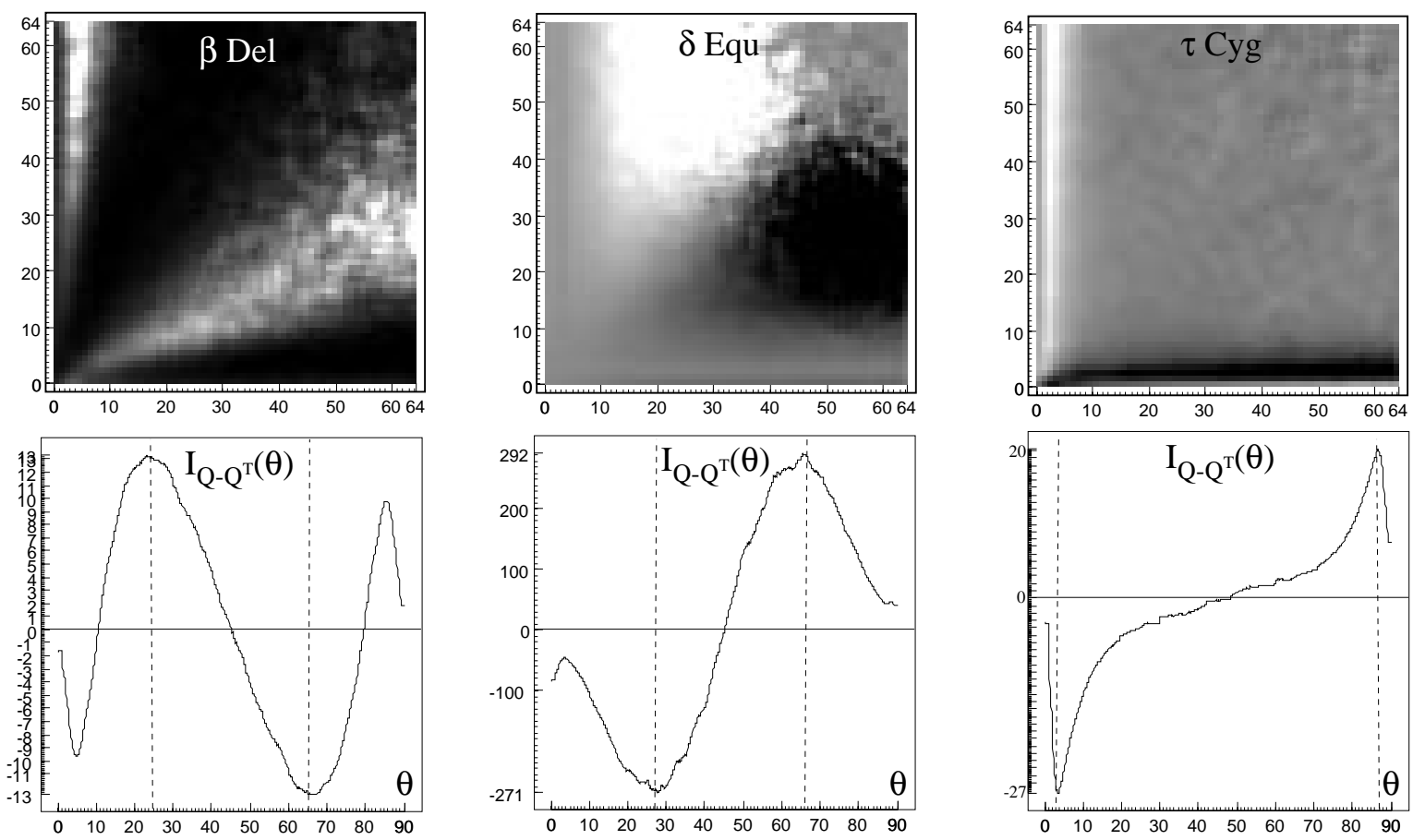

Fig. 3. Gray-level plots of function $Q-Q^{T}$, and plots of its radial integration $I_{Q-Q^{T}}$, computed on the specklegrams of three binary stars. The radial integrations show two extrema. The maximum is reached for $\arctan (\alpha)$, the minimum for $\arctan (1 / \alpha)$, $\alpha$ being the intensity ratio of the two binary components. A value of $\alpha<1$ means that the spatial lag vector goes from the brightest star to the lowest. The opposite if $\alpha>1$. This gives the absolute position angle of the couple 

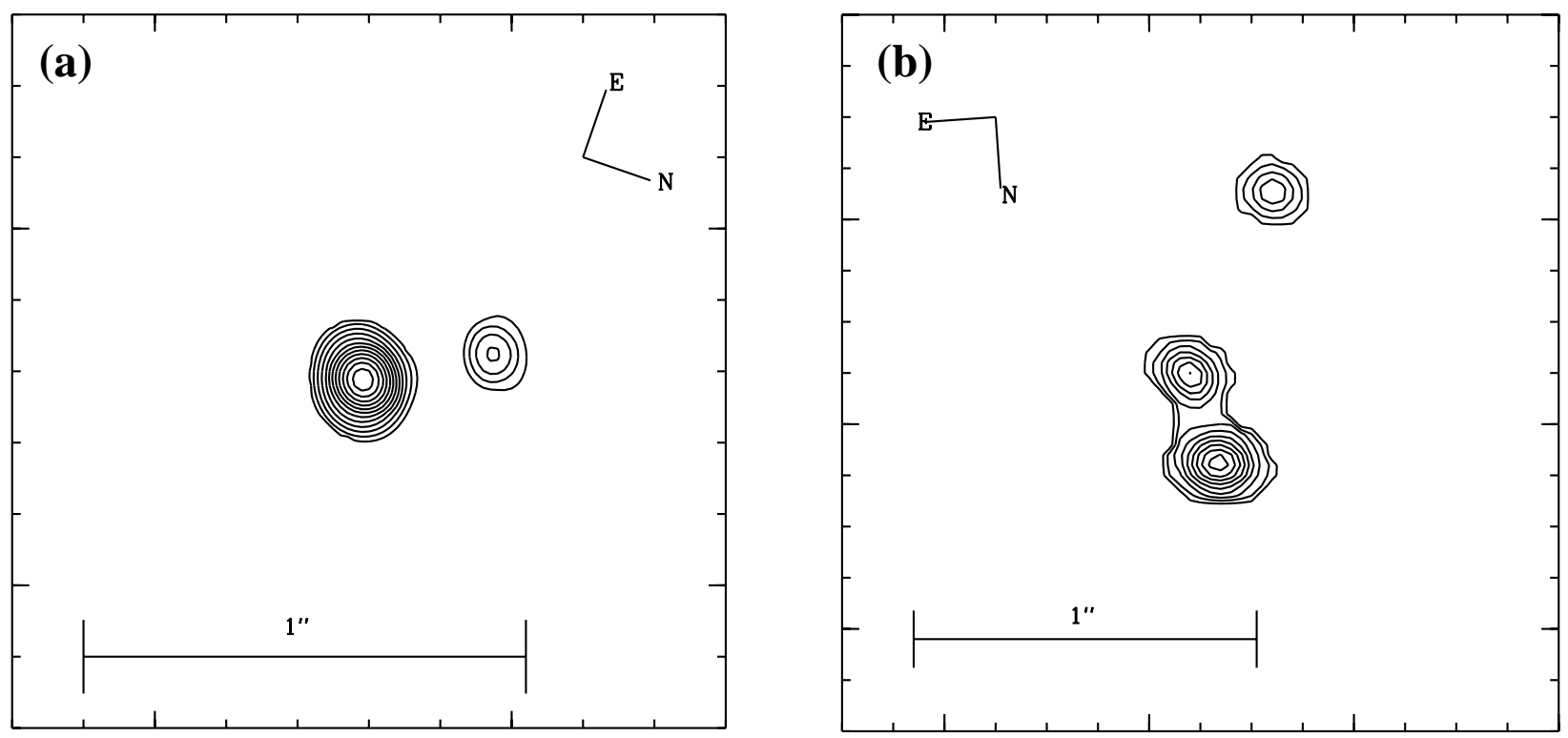

Fig. 4. Restored images of $\beta$ Del (a) and 2 Cam (b). These images were computed from 212 specklegrams (class 5 ) of $\beta$ Del and 1162 specklegrams (class 4 ) of 2 Cam. The image restoration procedure is described in Sect. 3.5

\subsection{Image restoration}

We have restored images of $\beta$ Del and 2 Cam (Fig. 4) using the bispectral method described in Lannes (1988) and Prieur et al. (1991).

The mean bispectrum and power spectrum were computed from the elementary frames of the same class (cf. Sect. 3.1). The phasor of the spectrum was derived from that of the mean bispectrum phasor through a global least-square minimization inversion method as described by Lannes (1988). This resulting phasor associated with the modulus of the spectrum derived from the mean power spectrum leads to an image which was then deconvolved by a Point Spread Function (PSF) obtained by observing a reference star (cf. Sect. 2). The deconvolution method we used (Lannes et al. 1987a, b) preserves the photometry, which allowed us to perform photometric analysis of the restored images. The measurements of the intensity ratios (taking the brightest component as a reference) lead to:

- for $\beta$ Del (ADS 14073):

$$
\Delta m(A B)=0.95 \pm 0.1
$$

- for 2 Cam (ADS 3358):

$$
\Delta m(A B)=0.58 \pm 0.1 \quad \Delta m(A C)=1.13 \pm 0.1
$$

which is in very good agreement with the measurements obtained with the twofold probability density functions described in Sect. 3.4 which are summarized in Table 2.

\section{Results and discussion}

The measurements are summarized in Table 2. Positions are compared with those computed from the latest available orbits. Orbital elements are from the catalog of Worley \& Heintz (1983) and the Sky Catalogue 2000 Vol. 2 for orbits prior to 1984. Recent orbital elements were found on the home page of Pr. W. Heintz (http://laser.swarthmore.edu/html/ research/heintzr.html) and in the literature (references are given in Col. 2).

This paper presents the first extensive application of speckle techniques based on the probability density functions of specklegrams. This method developed at Nice university is well adapted to the data reduction of binary star speckle data. Relative photometry computed this way (cf Sect. 3.4) is found to be consistent with direct image reconstruction from bispectral techniques (cf Sect. 3.5).

The residuals in $\rho$ and $\theta$ are given in Fig. 5. The cloud of points around $(\Delta \rho, \Delta \theta)=(0,0)$ validates the data reduction. It can however be noticed that several stars are found far from the expected position computed from the latest available orbit. Clearly some published orbits have to be reconsidered. This is the case for ADS 3711 (orbit estimated in 1969, period $P=199 \mathrm{y}$ ), ADS 4115 (1951, $P=586 \mathrm{y}), \operatorname{ADS} 5925(1966, P=512 \mathrm{y})$ and ADS 8035 (Couteau Orbit: 1958, $P=44.7 y$ (Sky Cat. 2000) - Heintz orbit: $1956, P=44.4 y$ (Worley \& Heintz 1983)).

The stars ADS 5447 and ADS 14073 are also found far from their expected position, although the orbits are quite recent (1989 for ADS 14073, 1992 for ADS 5447). More observations are clearly needed before giving a conclusion.

For the star ADS 2616, we have computed the position from the ephemeris of Luyten (1934) and Scardia (1985). Surprisingly, we found that the 1934 orbit does better fit the observed position. 
Table 2. Table of measurements. Predicted values of $\rho$ and $\theta$ are computed from the latest orbits available. Two orbits were given by Heintz for ADS 1860. Two orbits are also given for ADS 8035 because the latest one does not fit the measured position. Magnitude differences were measured with the probability density functions and are given in the color bands of Table 1

\begin{tabular}{|c|c|c|c|c|c|c|}
\hline $\begin{array}{c}\text { ADS } \\
\text { number }\end{array}$ & $\begin{array}{c}\text { Latest } \\
\text { orbit }\end{array}$ & $\begin{array}{c}\rho_{\text {obs }} \\
\left({ }^{\prime \prime}\right)\end{array}$ & $\begin{array}{c}\rho_{\text {cal }} \\
\left({ }^{\prime \prime}\right)\end{array}$ & $\begin{array}{c}\theta_{\text {obs }} \\
\left({ }^{\circ}\right)\end{array}$ & $\begin{array}{c}\theta_{\text {cal }} \\
\left({ }^{\circ}\right.\end{array}$ & $\Delta m_{\text {obs }}$ \\
\hline \hline 434 & Heintz 1995 & $0.440 \pm 0.015$ & 0.463 & $192.5 \pm 0.5$ & 193.2 & $0.14 \pm 0.05$ \\
\hline 1615 & Scardia 1983 & $1.99 \pm 0.03$ & 1.847 & $276 \pm 1$ & 274.3 & $0.095 \pm 0.090$ \\
\hline 1860 & Heintz 1996 & $3.018 \pm 0.010$ & 2.55 & $231 \pm 1$ & 231.9 & $1.67 \pm 0.06$ \\
& Heintz 1996 & & 2.555 & & 231.7 & $1.67 \pm 0.06$ \\
\hline 2616 & Luyten 1934 & $0.700 \pm 0.030$ & 0.732 & $1 \pm 1$ & 1.328 & - \\
& Scardia 1985 & & 0.640 & & 356.5 & \\
\hline $2755 \mathrm{AB}$ & Wierzbinsky 1956 & - & 0.118 & - & 83.12 & - \\
\hline $3358 \mathrm{AB}$ & Hartkopf et al. 1996 & $0.245 \pm 0.010$ & 0.258 & $170.0 \pm 0.5$ & 170.3 & $0.55 \pm 0.10$ \\
$3358 \mathrm{AC}$ & & $0.830 \pm 0.030$ & $0.713^{*}$ & $204.0 \pm 0.5$ & $204.5 *$ & $1.30 \pm 0.15$ \\
$3358 \mathrm{BC}$ & & $0.585 \pm 0.020$ & & $212.5 \pm 0.8$ & & $0.95 \pm 0.20$ \\
\hline 3711 & Baize 1969 & $0.76 \pm 0.13$ & 0.743 & $323 \pm 1$ & 331.3 & - \\
\hline 3728 & Muller 1963 & - & 0.218 & - & 357.0 & - \\
\hline 4115 & Siegrist 1951 & $1.090 \pm 0.005$ & 1.030 & $47.2 \pm 0.5$ & 40.92 & $1.55 \pm 0.20$ \\
\hline 5447 & Baize 1992 & $0.345 \pm 0.045$ & 0.387 & $218 \pm 1$ & 221.1 & - \\
\hline 5925 & Mourao 1966 & $0.600 \pm 0.015$ & 0.727 & $281.6 \pm 1.3$ & 282.5 & - \\
\hline 7307 & Arend 1953 & $1.017 \pm 0.005$ & 1.038 & $279.6 \pm 0.5$ & 281.7 & $0.25 \pm 0.15$ \\
\hline 8035 & Couteau 1958 & $0.565 \pm 0.015$ & 0.623 & $233 \pm 1$ & 240.9 & $1.78 \pm 0.09$ \\
& Heintz 1956 & & 0.435 & & 225.8 & \\
\hline 10360 & Hartkopf et al. 1989 & $0.132 \pm 0.005$ & 0.138 & $289.3 \pm 0.5$ & 108.0 & $0.05 \pm 0.10$ \\
\hline 12973 & Hartkopf et al. 1989 & $0.226 \pm 0.005$ & 0.230 & $157.5 \pm 0.5$ & 157.3 & $0.65 \pm 0.15$ \\
\hline 14073 & Hartkopf et al. 1989 & $0.220 \pm 0.005$ & 0.199 & $287 \pm 1$ & 278.9 & $0.88 \pm 0.06$ \\
\hline 14773 & Hartkopf et al. 1996 & $0.270 \pm 0.015$ & 0.270 & $27.65 \pm 0.5$ & 27.5 & $0.83 \pm 0.08$ \\
\hline 14787 & Heintz 1970 & $0.787 \pm 0.010$ & 0.733 & $337.8 \pm 0.5$ & 340.3 & $3.00 \pm 0.05$ \\
\hline 15281 & Hartkopf et al. 1989 & $0.086 \pm 0.005$ & 0.088 & $193.5 \pm 0.5$ & 199.2 & $0.08 \pm 0.08$ \\
\hline 16057 & Heintz 1991 & $0.250 \pm 0.015$ & 0.255 & $110 \pm 1$ & 111.1 & $0.1 \pm 0.1$ \\
\hline
\end{tabular}

*: Orbit of 2 Cam ABxC. $\dagger: 2$ orbits proposed.

\begin{tabular}{|c|c|l||r|r|r|}
\hline ADS & \multicolumn{1}{|c|}{$\Delta \rho$} & $\Delta \theta$ & ADS & \multicolumn{1}{|c|}{$\Delta \rho$} & \multicolumn{1}{|c|}{$\Delta \theta$} \\
\hline 434 & -0.023 & -0.7 & $8035^{3}$ & -0.058 & -7.9 \\
1615 & 0.143 & 1.7 & $8035^{4}$ & 0.130 & 7.2 \\
$1860^{1}$ & 0.468 & -0.9 & 10360 & -0.006 & 181.3 \\
$1860^{2}$ & 0.463 & -0.7 & 12973 & -0.004 & 0.2 \\
$2616^{5}$ & -0.032 & -0.328 & 14073 & 0.021 & 8.1 \\
$3358 \mathrm{AB}$ & -0.007 & -0.3 & 14773 & 0.000 & 0.15 \\
3711 & 0.17 & -8.3 & 14787 & 0.054 & 2.5 \\
4115 & 0.06 & 6.28 & 15281 & -0.002 & -5.7 \\
5447 & -0.042 & -3.1 & 16057 & -0.005 & -1.1 \\
5925 & -0.127 & -0.9 & & & \\
7307 & -0.021 & -1.9 & & & \\
\hline
\end{tabular}

${ }^{1}:$ st orbit given by Heintz.

${ }^{2}$ : 2nd orbit given by Heintz.

${ }^{3}$ : Couteau 1958. ${ }^{4}$ : Heintz 1956

${ }^{5}$ : Luyten 1934.

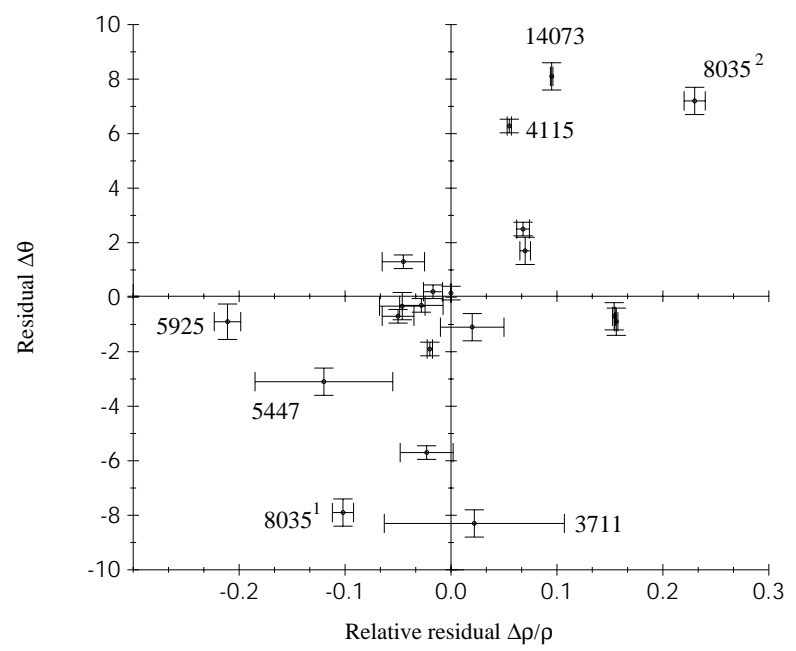

Fig. 5. Table on the left gives the residuals in separation $(\Delta \rho)$ and position angle $(\Delta \theta)$. Figure on the right is a graphics visualization of the residuals in a $(\Delta \rho / \rho, \Delta \theta)$ plane. Each measurement corresponds to a point in the graph. Error bars in $\rho$ and $\theta$ have been drawn to scale. For $c$ Her (ADS 10360) the residual in $\theta$ has been taken as $1.3^{\circ}$ for the plot (instead of $181.3^{\circ}$ ) 
The case of $c$ Her (ADS 10360) is interesting since we found a residual of $181.3^{\circ}$ on the position angle resulting from a confusion between components $\mathrm{A}$ and $\mathrm{B}$. This may occur when the magnitude difference is close to zero: measurements of $c$ Her available at the CHARA catalog (http://www.chara.gsu.edu/CHARA/chara.html) show sometimes a $180^{\circ}$ uncertainty on $\theta$ between successive observations. One could object that our observation was made in the red domain, but the spectral types of the two stars are identical (A9 IV) (which should be further investigated), and the brighter star of the couple is the same whatever the color. ADS 10360 needs to be observed again in several color bands for confirmation and to check whether the problem comes from a poor observation or a difference of spectral type of the two binary components.

Acknowledgements. The authors whish to thank the technical staff of the TBL: André Augé, Christian Decha, Pierre Déréthé, Christian Duthu, Francis Laccassagne, Jean-Marie LavieCambo and Christian Lucuix (Observatoire Midi-Pyrénées) for help during the observations; Michel Aurière (Observatoire Midi-Pyrénées) and Institut National des Sciences de l'Univers for financial support. Thanks are also due to Pr. Wulff Heintz for helping in using the orbital elements from his database. This work made use of the SIMBAD astronomical database and of the CHARA 3rd catalogue of interferometric measurements of binary stars.

\section{References}

André C., Festou M.C., Koechlin L., Lannes A., Pérez J.P., Prieur J.L., Roques S., 1994, Planet. Space Sci. 42, 747

Aristidi E., Carbillet M., Lyon J.-F., Aime C., 1997 (to be published in A\&AS)

Baize P., 1992, A\&AS 92, 31

Bagnuolo W.G Jr., 1988, Opt. Lett. 13, 907

Bagnuolo W.G. Jr., Mason B.D., Barry D.J., Hartkopf W.J.,
McAllister H.A., 1992, AJ 103, 1399

Carbillet M., Lopez B., Aristidi E., et al., 1996a, A\&A 314, 112

Carbillet M., Aristidi E., Aime C., Ricort G., 1996b (submitted to $A \& A)$

Fried D.L., 1966, J. Opt. Soc. Am. 56, 1372

Hartkopf W.I., McAllister H.A., Franz O.G., 1996, AJ 111, 370

Hartkopf W.I., Franz O.G., McAllister H.A., 1989, AJ 98, 1014

Heintz W.D., 1991, A\&AS 90, 311

Heintz W.D., 1995, ApJS 99, 693

Heintz W.D., 1996, ApJ 111, 408

Sky Catalogue 2000.0 Vol. 2, 1985, Hirshfeld A. and Sinnot R.W. (eds.)

Labeyrie A., 1970, A\&A 6, 85

Lannes A., Roques S., Cazanove M.J., 1987a, J. Mod. Opt. 34, 161

Lannes A., Roques S., Cazanove M.J., 1987b, J. Mod. Opt. 34, 321

Lannes A., 1988, Proc. of the NOAO-ESO conference on High Resolution Imaging by interferometry. Garching, Germany, p. 169

Lohman A.W., Weigelt G., Wirnitzer B., 1983, Appl. Opt. 22, 4028

Prieur J.-L., Lannes A., Cullum M., 1991, Proc. of the ESO conference on High Resolution Imaging by interferometry II. Garching, Germany, p. 353

Prieur J.-L., Festou M.C, Koechlin L., André C., 1994, Coll. National de Planétologie de l'INSU, Toulouse, S16

Roddier F., 1981, Progress in optics XIX, 282, Noth-Holland Pub. Co.

Roddier F., 1988, Physics Report (review section of Physics Letters) 170,97

Scardia M., 1985, A\&AS 59, 455

Weigelt G., 1991, Progress in optics, XXIX, 293, Noth-Holland Pub. Co.

Worley C.E., Heintz W.D., 1983, Pub. U.S. Naval Obs. (2) 24, part VII (available at SIMBAD:

http://simbad.u-strasbg.fr/cgi-bin/cdsbib/) 